\title{
The Impacts of Swimming Exercise on Hippocampal Expression of Neurotrophic Factors in Rats Exposed to Chronic Unpredictable Mild Stress
}

\author{
Pei Jiang, Rui-Li Dang, Huan-De Li, Li-Hong Zhang, \\ Wen-Ye Zhu, Ying Xue, and Mi-Mi Tang \\ Institute of Clinical Pharmacy \& Pharmacology, Second Xiangya Hospital, Central South University, Changsha 410011, China \\ Correspondence should be addressed to Huan-De Li; lihuande1953@126.com
}

Received 24 June 2014; Accepted 14 August 2014; Published 27 October 2014

Academic Editor: David Mischoulon

Copyright (C) 2014 Pei Jiang et al. This is an open access article distributed under the Creative Commons Attribution License, which permits unrestricted use, distribution, and reproduction in any medium, provided the original work is properly cited.

Depression is associated with stress-induced neural atrophy in limbic brain regions, whereas exercise has antidepressant effects as well as increasing hippocampal synaptic plasticity by strengthening neurogenesis, metabolism, and vascular function. A key mechanism mediating these broad benefits of exercise on the brain is induction of neurotrophic factors, which instruct downstream structural and functional changes. To systematically evaluate the potential neurotrophic factors that were involved in the antidepressive effects of exercise, in this study, we assessed the effects of swimming exercise on hippocampal mRNA expression of several classes of the growth factors (BDNF, GDNF, NGF, NT-3, FGF2, VEGF, and IGF-1) and peptides (VGF and NPY) in rats exposed to chronic unpredictable mild stress (CUMS). Our study demonstrated that the swimming training paradigm significantly induced the expression of BDNF and BDNF-regulated peptides (VGF and NPY) and restored their stress-induced downregulation. Additionally, the exercise protocol also increased the antiapoptotic Bcl-xl expression and normalized the CUMS mediated induction of proapoptotic Bax mRNA level. Overall, our data suggest that swimming exercise has antidepressant effects, increasing the resistance to the neural damage caused by CUMS, and both BDNF and its downstream neurotrophic peptides may exert a major function in the exercise related adaptive processes to CUMS.

\section{Introduction}

Depression is a debilitating and widely distributed disorder which is associated with exposure to stressful life events. Studies of chronic stress in animal models and postmortem tissues from depressed patients demonstrated that reduced size of limbic brain regions that regulate mood and cognition and decreased neuronal synapses in these brain areas may contribute to the pathogenesis of depression [1]. There is emerging evidence that exercise has antidepressant effects, whereby promoting neurogenesis and inhibiting neurodegeneration [2]. Although exercise seems to have therapeutic and preventive effects on the course of depression, the underlying mechanisms remain elusive. It has been proposed that the key mechanism mediating the broad benefits of exercise on the brain is induction of neurotrophic factors, which instruct downstream structural and functional changes [3]. The protective effects of exercise from chronic stress have been best-studied in the hippocampus, where exercise increased synaptic plasticity and neurotrophic factors expression. Previous studies indicate that exercise can promote hippocampal neurotrophic cascades and enhance neural survival, differentiation, connectivity, and plasticity, while stress shows the opposite effects, which indicates a potential mechanism for exercise to alleviate stress [4].

Brain-derived neurotrophic factor (BDNF) is the most abundantly expressed neurotrophin in the mature central nervous system and supports the survival of many types of neurons. A number of animal studies have documented that the exposure to chronic stress can result in decreased BDNF expression in hippocampus [5]. Conversely, both antidepressant treatment and exercise can enhance hippocampal BDNF status $[6,7]$. The neuropeptide VGF and neuropeptide Y (NPY) have been implicated in the actions of BDNF and 
both of which can be induced by BDNF and antidepressants $[8,9]$. Accumulating evidence has shown that BDNF was implicated in the pathophysiology of depression and the antidepressant action of exercise. Nevertheless, the role of its downstream neuropeptides and other neurotrophic factors remains unclear. Other neurotrophins, including nerve growth factor (NGF), glia cell-derived neurotrophic factor (GDNF), and neurotrophin-3 (NT-3), are also important factors for regulation of neuroplasty and were implied to play a role in the neurotrophic hypothesis of depression [10]. There are several additional growth factors that also have been implicated in neurogenesis, depression, and treatment response, such as insulin-like growth factor-1 (IGF-1), vascular endothelial growth factor (VEGF), and fibroblast growth factor-2 (FGF-2) [11].

It was reported that swimming exercise could reverse the chronic unpredictable mild stress (CUMS) induced depression-like state in rodents [12-14]. However, the results are inconsistent and the underlying mechanisms are far from fully understood [15]. Since neurotrophic factors are suggested to exert a major function in the antidepressant effects of exercise, the main objective of the present study was to further establish the therapeutic role of swimming exercise in depression and systematically evaluate the potential neurotrophic factors that were involved in the antidepressive effects of the exercise paradigm. The expression of biomarkers of cell survival including the antiapoptotic protein $\mathrm{Bcl}-\mathrm{xl}$ and the proapoptotic protein Bax was also assessed [16].

\section{Materials and Methods}

2.1. Animals. Experiments were carried out with male Sprague-Dawley rats (250-280 g), supplied by the Experimental Animal Center of the Second Xiangya Hospital. The rats were housed at $22-25^{\circ} \mathrm{C}$ and humidity $50-60 \%$ with a $12 \mathrm{~h}$ light-dark cycle and had free access to commercial rat chow and water, except when they were submitted to CUMS. All animal use procedures were carried out in accordance with the Regulations of Experimental Animal Administration issued by the State Committee of Science and Technology of the People's Republic of China, with the approval of the Ethics Committee in our university.

2.2. CUMS Procedure and Exercise Protocol. The rats were randomly divided into four groups $(n=8)$ : Control group, Exercised group, Stressed group, and Stressed + Exercised group. While the rats in Control group were undisturbed, the Exercised group was trained in a progressively increasing moderate swimming protocol as previously reported with minor change [13]. Swimming exercise was performed in a plastic water tank $(100 \mathrm{~cm} \times 80 \mathrm{~cm} \times 90 \mathrm{~cm})$ at $32 \pm 1^{\circ} \mathrm{C}$ and a depth of $55 \mathrm{~cm}$. The protocol included two phases: adaptation and training. In the adaptive phase, the rats swam $20 \mathrm{~min}$ per day for 6 days. The adaptation was aimed at reducing the water induced stress without promoting physiological alterations in relation to the physical training [17]. After rest for one day, the training period began from the first day of the second week. Swimming duration was progressively increased from $20 \mathrm{~min}$ to $70 \mathrm{~min}$ per day for 6 days in 1 week. This intensity was maintained to the end of the training program. The swimming program lasted 6 days per week for a total of 4 weeks. The beneficial effects of the swimming protocol have been repeatedly validated by previous reports $[13,18]$.

The Stressed group received a previously established weekly stress regime with minor modification [19]. Briefly, the CUMS rats were randomly exposed to one of these stressors per day for 4 weeks and the same stressor was not used in 2 consecutive days: cage tilting $\left(45^{\circ}\right)$ for $24 \mathrm{~h}$; water deprivation for $24 \mathrm{~h}$, finally with $1 \mathrm{~h}$ an empty bottle; $1 \mathrm{~min}$ tail clamping; physical restraint for 2 hours; fasting for $24 \mathrm{~h}$; soiled cage for $24 \mathrm{~h}$; and $20 \mathrm{~min}$ noise. The Stress + Exercised rats received both CUMS and the exercise protocol. As isolation may cause additional stress to the animals [20], the Stressed and Stressed + Exercised group were housed separately (cage size: $26 \times 19 \times 15 \mathrm{~cm}$ ), while four rats in the other two groups shared one cage (cage size: $90 \times 45 \times 25 \mathrm{~cm}$ ).

2.3. Sucrose Preference Test (SPT). SPT is a measure of stressed-induced anhedonia state, a key depressive-like behavior in rats [19]. Prior to SPT, all the rats were housed individually and habituated to $48 \mathrm{~h}$ of forced $1 \%$ sucrose solution consumption in two bottles on each side. Then after $16 \mathrm{~h}$ water deprivation, we placed two preweighted bottles, one containing $1 \%$ sucrose solution and another containing tap water, to each rat. The side (left and right) of the two bottles was randomly placed, in order to avoid spatial bias. The bottles were weighted again after $1 \mathrm{~h}$ and the weight difference was considered to be the rat intake from each bottle. The preference for sucrose was measured as a percentage of the consumed $1 \%$ sucrose solution relative to the total amount of liquid intake.

2.4. Open Field Test (OFT). OFT is a measure to evaluate the general locomotion and exploratory behavior of rats [12]. As previously described, the test was conducted between 8:00 and 12:00, one day after SPT. Each rat was placed at the centre of an apparatus with a square arena $(90 \mathrm{~cm} \times 90 \mathrm{~cm} \times 40 \mathrm{~cm})$. The floor of the arena was equally divided into 25 squares. The locomotor activity was video-taped and the time in the centre square and number of crossings (squares of crossings with all paws) were manually counted for $5 \mathrm{~min}$. Between the tests, the apparatus was thoroughly cleaned and dried.

2.5. Real-Time PCR Analysis. Total RNA from hippocampus was isolated using Trizol reagent (Invitrogen, USA) according to the manufacturer's instructions. Quantification of mRNAs was performed on Bio-rad Cx96 Detection System (Bio-rad, USA) using SYBR green PCR kit (Applied Biosystems, USA) and gene-specific primers. Each cDNA was tested in triplicate with 40 cycles of amplification. Relative quantitation for PCR product was normalized to $\beta$-actin as internal standard. The sequences of gene-specific primers are summarized in Table 1 .

2.6. Statistics. Statistical analysis was performed with SPSS 13.0 software. All values were presented as mean \pm SEM. Body 
TAble 1: Primer sequences used for the qPCR analysis.

\begin{tabular}{lllc}
\hline Gene (accession no.) & Sense primer $\left(5^{\prime}-3^{\prime}\right)$ & Antisense primer $\left(5^{\prime}-3^{\prime}\right)$ & Amplicon length \\
\hline BDNF (NM001270630) & TACCTGGATGCCGCAAACAT & GTAGAAATATTGCTTCAGTTGG & $200 \mathrm{bp}$ \\
GDNF (NM019139) & CAGAGGGAAAGGTCGCAGAG & CGTAGCCCAAACCCAAGTCA & $95 \mathrm{bp}$ \\
NT-3 (NM001270868) & GGGAGAGATCAAAACCGGC & TTGCGACGTTTTGCACT & $136 \mathrm{bp}$ \\
NGF (NM001277055) & ACATCAAGGGCAAGGAGG & GTGAGTCGTGGTGCAGTATG & $164 \mathrm{bp}$ \\
FGF-2 (NM019305) & TCCATCAAGGGAGTGTGTGC & TCCGTGACCGGTAAGTGTTG & $139 \mathrm{bp}$ \\
VEGF (NM001110333) & TATGTTTGACTGCTGTGGACTTGA & CAGGGATGGGTTTGTCGTGT & $204 \mathrm{bp}$ \\
IGF-1 (NM001082477) & TCAGTTCGTGTGTGGACCAG & CACAGCTCCGGAAGCAAC & $117 \mathrm{bp}$ \\
NPY (NM012614) & TGTTTGGGCATTCTGGCTGAGG & TTCTGGGGGCGTTTTCTGTGCT & $205 \mathrm{bp}$ \\
VGF (NM030997) & GATGAGTTGCCGGACTGG & CAACGCGTGATGGAAGTGAC & $159 \mathrm{bp}$ \\
Bcl-xl (NM001033670) & TGTGGCTGGTGTAGTTCTGC & CAGAAAAGCATTCCCGAGAG & $402 \mathrm{bp}$ \\
Bax $($ NM017059) & CTGCAGAGGATGATTGCTGA & GATCAGCTCGGGCACTTTAG & $174 \mathrm{bp}$ \\
$\beta$-Actin (NM031144) & CATCCTGCGTCTGGACCTGG & TAATGTCACGCACGATTTCC & $116 \mathrm{bp}$ \\
\hline
\end{tabular}

weight gain was analyzed using a two-way repeated ANOVA analysis with Stress and Exercise as between subject factors and time as within subject factor. The data of SPT, OFT, and relative mRNA expression were analyzed by using twoway ANOVA followed by Fisher's least significant difference (LSD) post hoc test. The prior level of significance was established at $P<0.05$.

\section{Results}

3.1. Behavioral Test and Body Weight Gain. Both exercise and CUMS procedure significantly slowed down the body weight growing rate (Figure 1(a)). Stress exposure induced a significant decrease in sucrose preference. Exercise had no effect on sucrose intake in nonstressed rats, but significantly reversed the decline in the sucrose preference of CUMS rats (Figure 1(b)). The locomotor activity and exploratory behavior were observed in the OFT (Figures 1(c) and 1(d)). 4 weeks of CUMS led to a significantly decreased number of crossings and increased time spent in centre. The Exercised group seems to be more explorative, with less time spent in centre and more numbers of crossings. The Stressed + Exercised group also showed improved exploratory behavior compared with Stressed group. Overall, the results suggest that swimming exercise can successfully ameliorate the stress-induced depression-like state in rats.

3.2. Neurotrophic Effects of Swimming Exercise. Since neurotrophic factors play a major role in the antidepressant action of exercise, we assessed the expression of the neurotrophic factors that related to depression in the hippocampus. As previously reported [21], exercise significantly induced the expression of BDNF, the mostly documented biomarker in depression, and reversed the inhibitory effects of chronic stress (Figure 2(a)). Interestingly, stress induced GDNF (Figure 2(b)) and NT-3 (Figure 2(c)) expression, while no influence of exercise on the two neurotrophins was found, which suggests a potential compensatory adaptative mechanism to CUMS. Both NGF (Figure 2(d)) and FGF2 (Figure 2(e)) were downregulated by CUMS, while the swimming protocol also had no effect on their expression.
Hippocampal generation of VEGF (Figure 2(f)) and IGF1 (Figure 2(g)) was not influenced by chronic stress, but exercise significantly increased the expression of IGF-1 in non-CUMS rats. In parallel to the expression of BDNF, the mRNA levels of its downstream neurotrophic peptides NPY (Figure 2(h)) and VGF (Figure 2(i)) were synchronously inhibited by stress and restored by exercise. Chronic stress also downregulated Bcl-xl mRNA status (Figure 3(a)) and upregulated the expression of Bax (Figure 3(b)). The stressinduced decreased ratio of $\mathrm{Bcl}-\mathrm{xl} / \mathrm{Bax}$ indicated that the neural cells were shifted toward cell death (Figure 3(c)). However, exercise was found to promote $\mathrm{Bcl}$-xl expression and increase the $\mathrm{Bcl}-\mathrm{xl} / \mathrm{Bax}$ ratio in non-CUMS rats. Meanwhile, in Stressed + Exercised group, the swimming protocol partly normalized the stress-induced imbalance between $\mathrm{Bcl}-\mathrm{xl}$ and Bax.

\section{Discussion}

Neural atrophy has been reported in rodent chronic stress models and clinical postmortem studies of depressed patients. These findings indicate that depression can be considered as mild neurodegenerative disorder, and the increased neural cell loss may contribute to the pathogenesis of depression [22]. Recent studies have strengthened the role of the abnormalities in neurotrophic pathways in the psychopathophysiology of depression. It has been repeatedly documented that chronic stress and depression are associated with the abnormal neurotrophic signaling in the brain [23]. Exercise is an effective strategy for depression treatment. A key mechanism mediating the antidepressant action of exercise is induction of central and periphery growth factor cascades. Several classes of neurotrophic factors are implicated in the neural atrophy of depression and beneficial effects of exercise. Although most work has focused on BDNF, other growth factors also play a role in the stress-induced neural cell loss.

Several lines of evidence from animal and human researches converge on the idea that BDNF is essential for hippocampal synaptic plasticity and modulation of depression. The exercise-evoked BDNF release has been repeatedly 


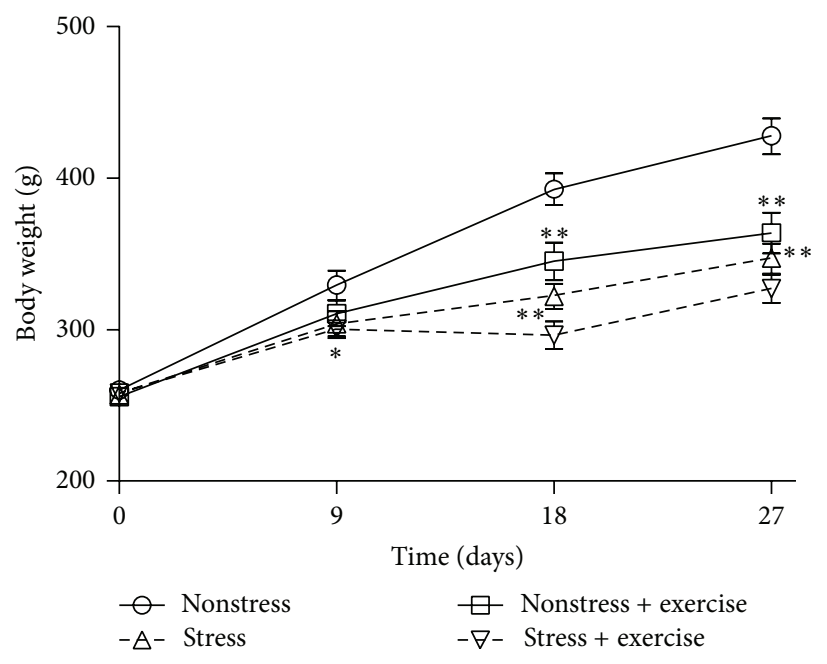

(a)

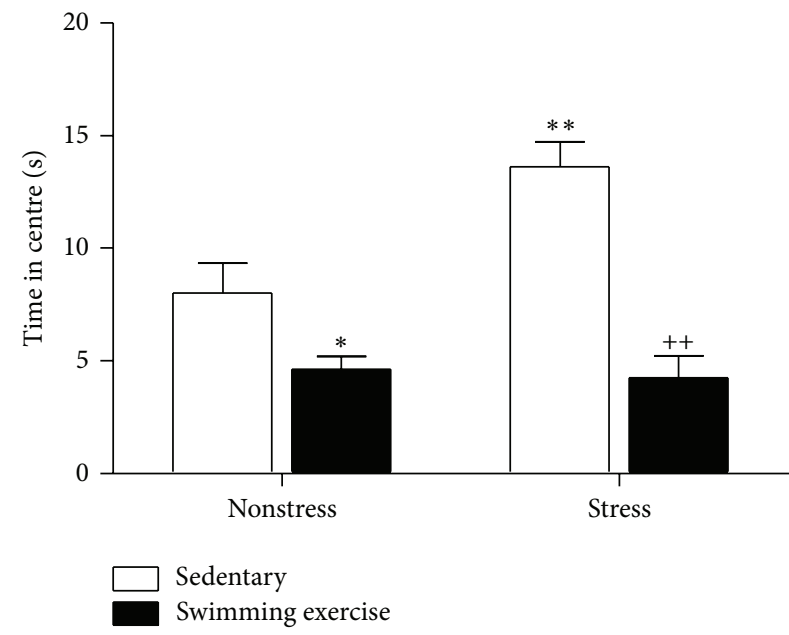

(c)

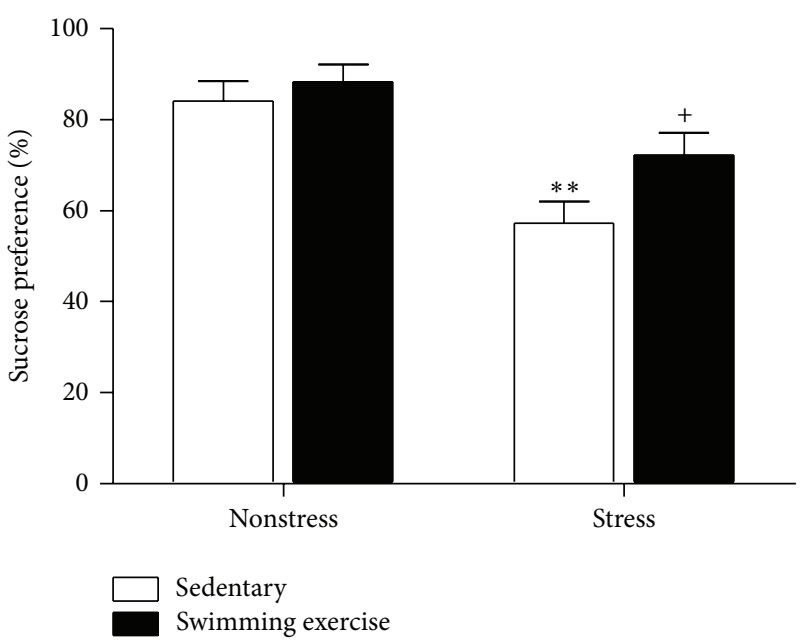

(b)

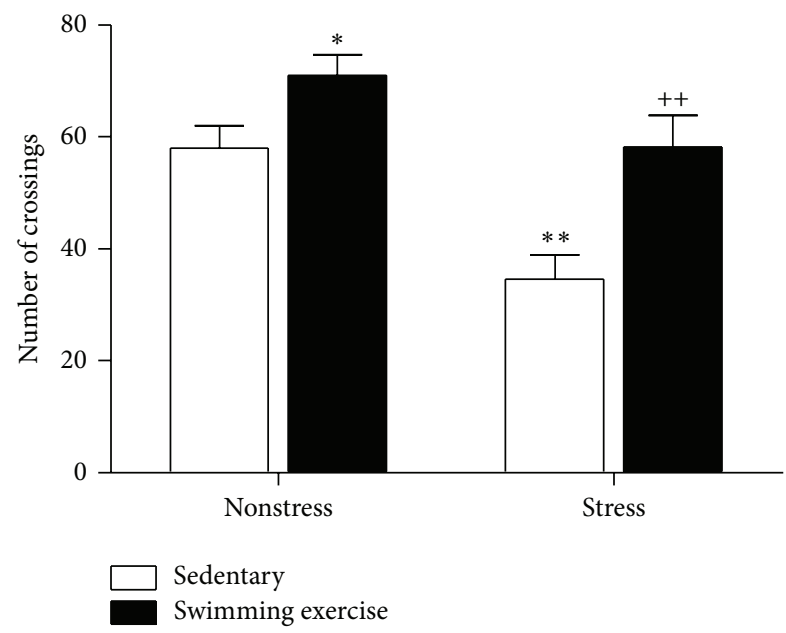

(d)

FIGURE 1: Effect of swimming exercise and CUMS on rat body weight (a) and behavioral changes in sucrose preference test (b) and time spent in centre (c) and number of crossings $(\mathrm{d})$ in open field test. Data are means $\pm \operatorname{SEM}(n=8) .{ }^{*} P<0.05,{ }^{* *} P<0.01$ compared to Control group. ${ }^{+} P<0.05,{ }^{++} P<0.01$ compared to Stressed group.

postulated to underlie the antidepressant effects of physical activity $[6,23,24]$. In the present study, we confirmed the antidepressant effects of the swimming protocol, and, as previously reported, the induction of BDNF expression was also found in this study, suggesting the involvement of BDNF in the amelioration of depression by swimming exercise. Interestingly, in parallel to BDNF, a synchronous rise of NPY and VGF was also found in the Exercised group. The mRNA levels of NPY and VGF were inhibited in Stressed group, but were normalized in the Stressed + Exercised group. Both NPY and VGF are related to neuronal synaptic remodeling and could be highly induced by BDNF in vitro and in vivo $[9,25]$. As NPY and VGF also actively participate in the neurotrophic hypothesis of depression and could be regulated by BDNF, our data argue that both BDNF and its downstream neurotrophic peptides, NPY and VGF, may play a role in the antidepressant effects of exercise.
Additionally, the effects of swimming exercise and stress exposure on other neurotrophic factors including GDNF, NT-3, NGF, and FGF-2 were also evaluated in this study. The exercise protocol had no influence on the four factors expression, but a significant reduction of NGF and FGF-2 was found in the Stressed group. While inhibiting NGF and FGF2 expression, it has been reported that exposure to chronic stress upregulated NT-3 and GDNF [26, 27]. However, the results were inconsistent $[28,29]$. In our study, we confirmed the increase of NT-3 and GDNF, which suggests a compensatory adaptive process to the stress-induced alterations of neurotrophic signaling in the hippocampus.

VEGF and IGF-1 are other two principle growth factors known to mediate the effects of exercise on the brain. Unlike the neurotrophins above, although both VEGF and IGF-1 can be synthesized in the brain, peripheral production is the main source of circulating VEGF and IGF-1. Both VEGF and 


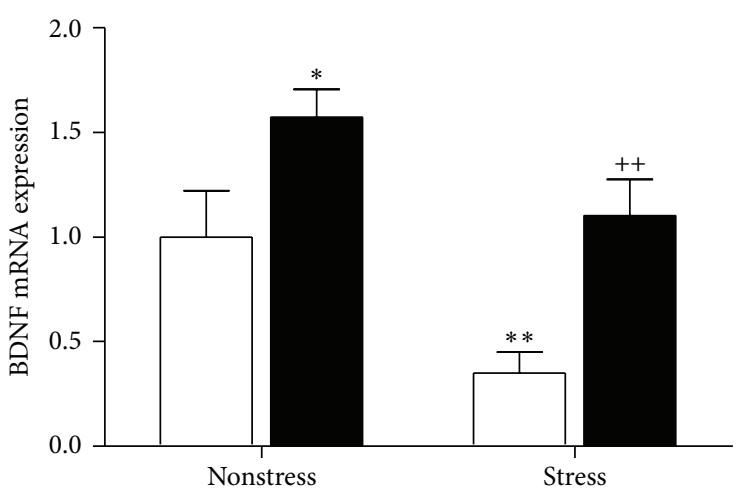

(a)

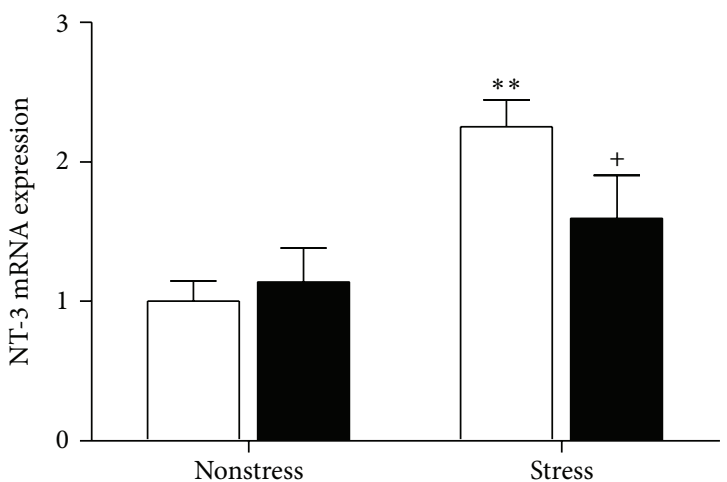

(c)

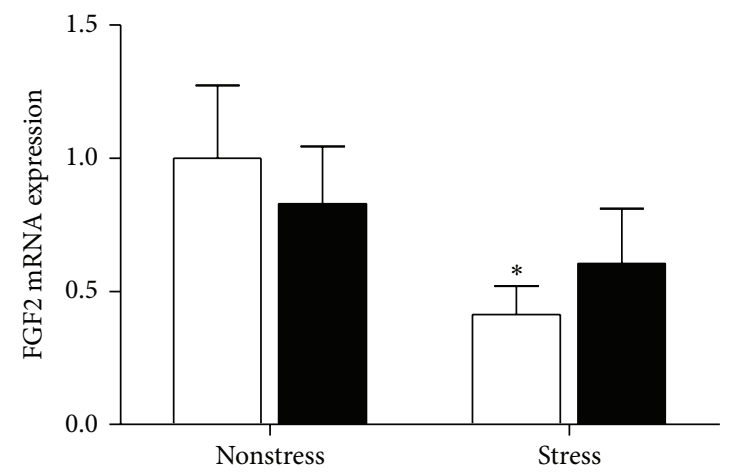

(e)

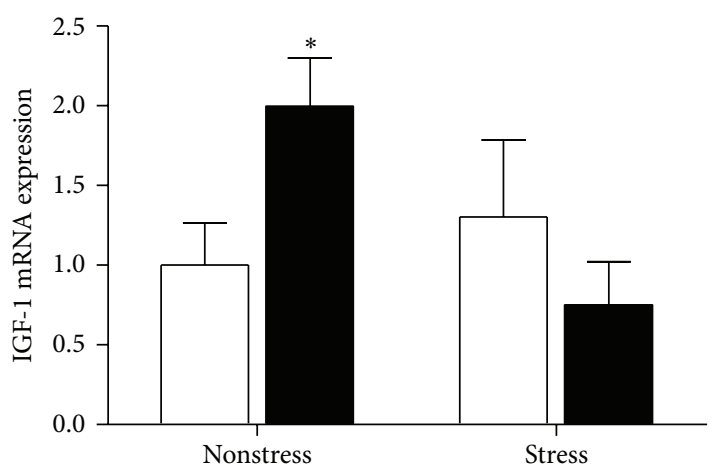

Sedentary

Swimming exercise

(g)

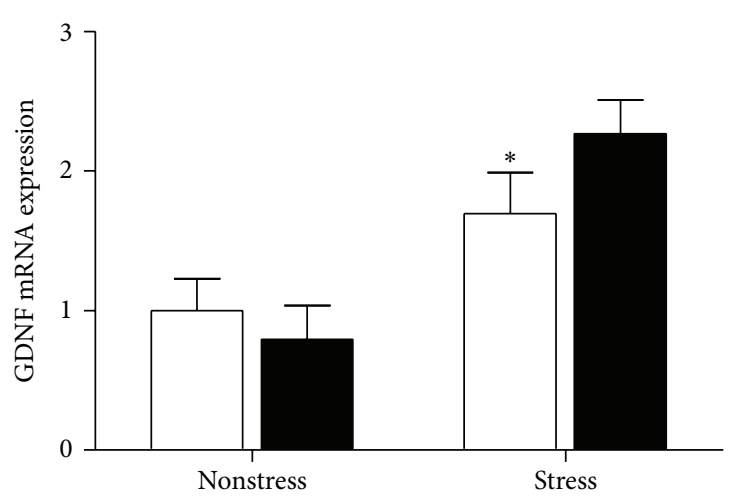

(b)

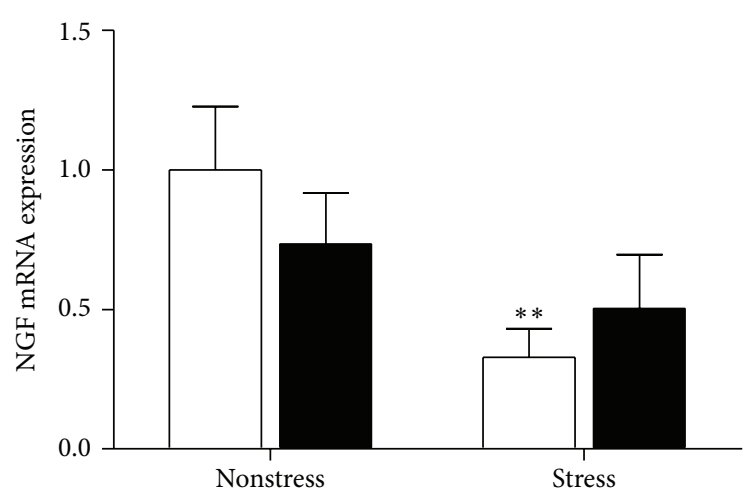

(d)

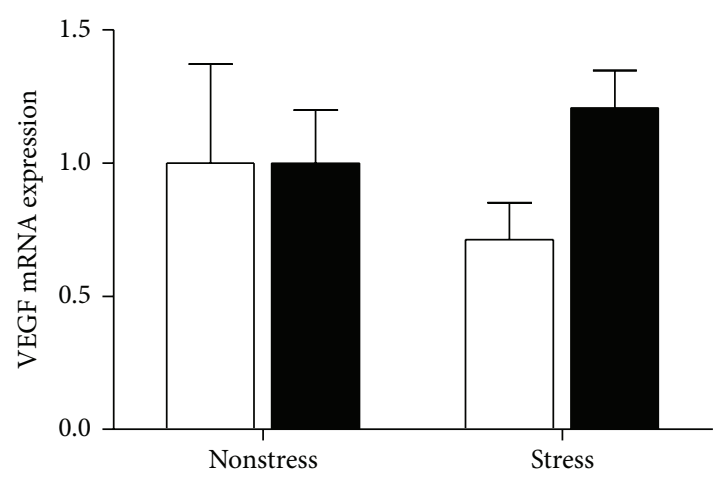

(f)

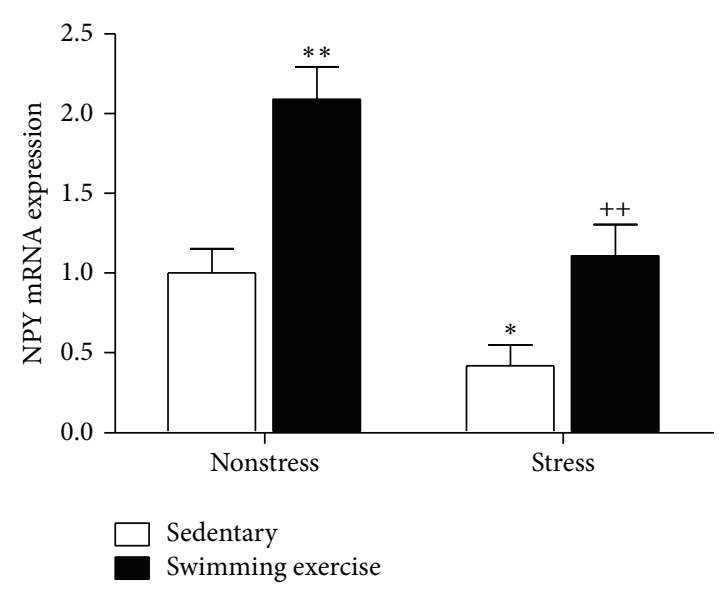

(h)

FIgUre 2: Continued. 


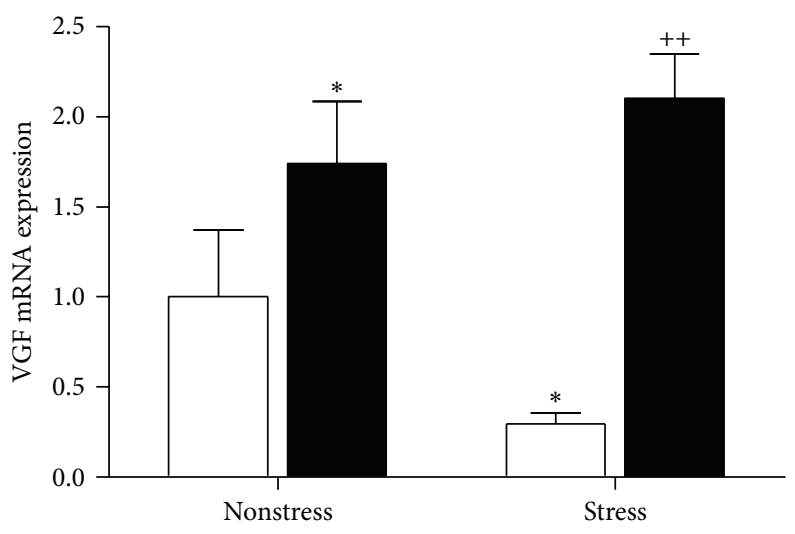

Sedentary

Swimming exercise

(i)

FIgURE 2: Effect of swimming exercise and CUMS on the neurotrophic factors expression. Data are means \pm SEM $(n=6) .{ }^{*} P<0.05$, ${ }^{* *} P<0.01$ compared to Control group. ${ }^{+} P<0.05,{ }^{++} P<0.01$ compared to Stressed group.

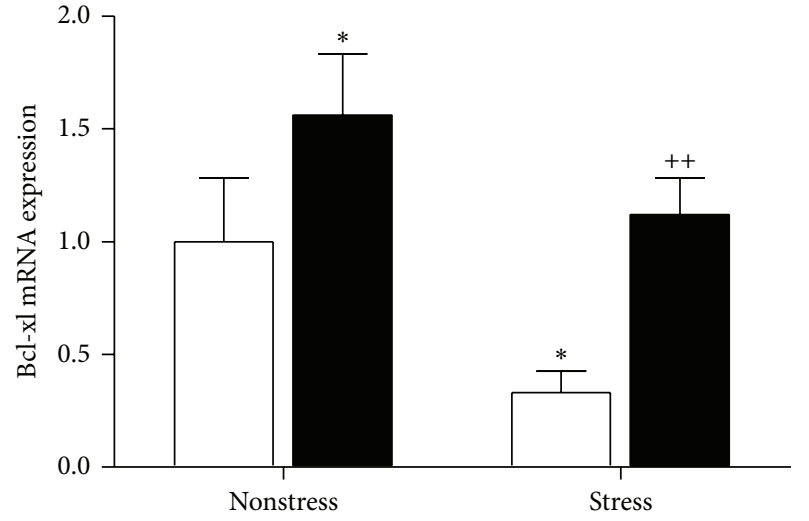

Sedentary

Swimming exercise

(a)

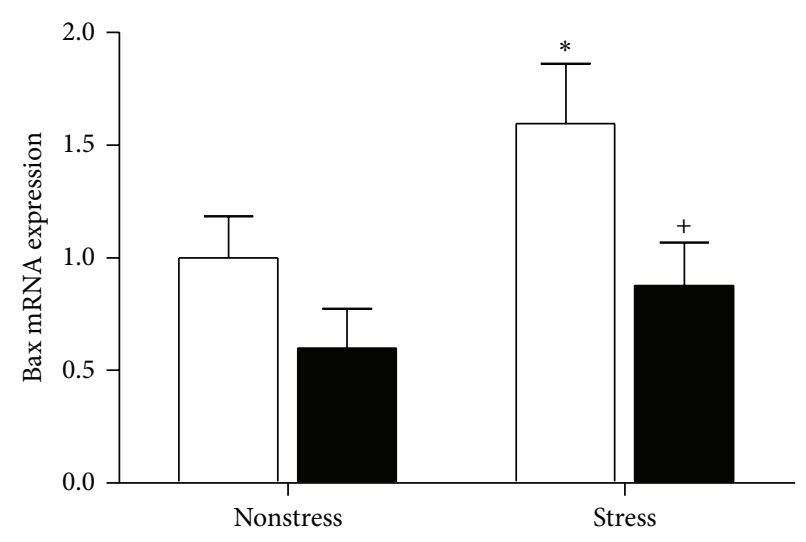

Sedentary

Swimming exercise

(b)

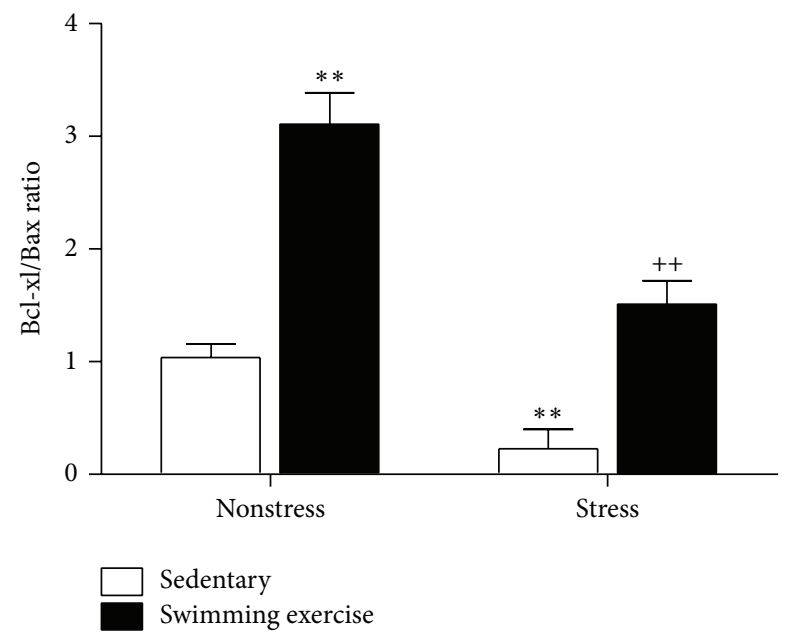

(c)

Figure 3: Effect of swimming exercise and CUMS on the expression of Bcl-xl (a) and Bax (b) and ratio of Bcl-xl/Bax (c). Data are means \pm $\operatorname{SEM}(n=6) .{ }^{*} P<0.05,{ }^{* *} P<0.01$ compared to Control group. ${ }^{+} P<0.05,{ }^{++} P<0.01$ compared to Stressed group. 
IGF-1 are essential for exercise stimulated neurogenesis, and blocking either VEGF or IGF-1 signaling prevents exerciseinduced neurogenesis in the hippocampus [30, 31]. However, the evidence of the influence of exercise on central generation of VEGF and IGF-1 is limited. In our study, we found that exercise only increased the hippocampal IGF-1 expression without any alterations of VEGF. Nevertheless, the significant change was not found between Stressed and Stressed + Exercised group, implying that the hippocampal production of IGF-1 does not play a key role in the antidepressant effects of swimming exercise. Since both VEGF and IGF1 are increased in the periphery by exercise and can cross the blood-brain-barrier to enter the brain [32, 33], our data indicate that the beneficial effects of exercise appear to be mediated by the periphery genesis of the two factors, but not through the influence on paracrine effects of VEGF and IGF-1 in the hippocampus.

The neural apoptosis process is programmed and controlled by the cellular balance between antiapoptotic Bcl$\mathrm{xl}$ and proapoptotic Bax expression. As previously reported, chronic stress downregulated the expression of $\mathrm{Bcl}-\mathrm{xl}$ and upregulated Bax status [34, 35]. The altered balance indicates that the neural cell is shifted toward apoptosis. The beneficial effect of swimming exercise on neural survival was further validated by the restored balance between Bcl-xl and Bax. The enhanced expression of Bcl-xl was also found in the non-CUMS rats after the exercise protocol, suggesting that swimming exercise can not only reverse the apoptotic effect of stress but also improve the basal neural cell survival.

\section{Conclusions}

Collectively, since the neurotrophic signaling can regulate neural survival and $\mathrm{Bcl}-\mathrm{xl} / \mathrm{Bax}$ expression, and the reversed effects of swimming exercise on the stressinduced neurotrophic alterations were only found in BDNF and its downstream peptides (NPY and VGF) expression, our data suggests that the neurotrophic benefits of swimming exercise in the hippocampus are mainly through the BDNF signaling pathway, and NPY and VGF also synergetically play a role in the antidepressant action of swimming exercise.

\section{Conflict of Interests}

The authors declare that there is no conflict of interests regarding the publication of this paper.

\section{Acknowledgments}

This research was supported by Hunan Provincial Innovation Foundation for Postgraduate (no. CX2013B099) and National Natural Science Foundation of China (no. 81101001).

\section{References}

[1] G. Masi and P. Brovedani, "The hippocampus, neurotrophic factors and depression: possible implications for the pharmacotherapy of depression," CNS Drugs, vol. 25, no. 11, pp. 913-931, 2011.
[2] H. Eyre and B. T. Baune, "Neuroimmunological effects of physical exercise in depression," Brain, Behavior, and Immunity, vol. 26, no. 2, pp. 251-266, 2012.

[3] M. Llorens-Martín, I. Torres-Alemán, and J. L. Trejo, "Growth factors as mediators of exercise actions on the brain," NeuroMolecular Medicine, vol. 10, no. 2, pp. 99-107, 2008.

[4] C. W. Cotman, N. C. Berchtold, and L.-A. Christie, "Exercise builds brain health: key roles of growth factor cascades and inflammation," Trends in Neurosciences, vol. 30, no. 9, pp. 464472, 2007.

[5] R. S. Duman and G. K. Aghajanian, "Synaptic dysfunction in depression: potential therapeutic targets," Science, vol. 338, no. 6103, pp. 68-72, 2012.

[6] L. Marais, D. J. Stein, and W. M. U. Daniels, "Exercise increases BDNF levels in the striatum and decreases depressive-like behavior in chronically stressed rats," Metabolic Brain Disease, vol. 24, no. 4, pp. 587-597, 2009.

[7] M. P. Mattson, "Energy intake and exercise as determinants of brain health and vulnerability to injury and disease," Cell Metabolism, vol. 16, no. 6, pp. 706-722, 2012.

[8] M. Heilig, "The NPY system in stress, anxiety and depression," Neuropeptides, vol. 38, no. 4, pp. 213-224, 2004.

[9] S. Thakker-Varia and J. Alder, "Neuropeptides in depression: role of VGF," Behavioural Brain Research, vol. 197, no. 2, pp. 262 278, 2009.

[10] W. N. Marsden, "Synaptic plasticity in depression: Molecular, cellular and functional correlates," Progress in NeuroPsychopharmacology and Biological Psychiatry, vol. 43, pp. 168184, 2013.

[11] M. Banasr, J. M. Dwyer, and R. S. Duman, "Cell atrophy and loss in depression: reversal by antidepressant treatment," Current Opinion in Cell Biology, vol. 23, no. 6, pp. 730-737, 2011.

[12] W. Liu and C. Zhou, "Corticosterone reduces brain mitochondrial function and expression of mitofusin, BDNF in depression-like rodents regardless of exercise preconditioning," Psychoneuroendocrinology, vol. 37, no. 7, pp. 1057-1070, 2012.

[13] X. Liu, L. J. Yang, S. J. Fan, H. Jiang, and F. Pan, "Swimming exercise effects on the expression of HSP70 and iNOS in hippocampus and prefrontal cortex in combined stress," Neuroscience Letters, vol. 476, no. 2, pp. 99-103, 2010.

[14] A. R. Sigwalt, H. Budde, I. Helmich et al., "Molecular aspects involved in swimming exercise training reducing anhedonia in a rat model of depression," Neuroscience, vol. 192, pp. 661-674, 2011.

[15] Y. Zhao, L.-J. Liu, C. Wang, and S.-X. Li, "Swimming exercise may not alleviate the depressive-like behaviors and circadian alterations of neuroendocrine induced by chronic unpredictable mild stress in rats," Neurology, Psychiatry and Brain Research, vol. 18, no. 4, pp. 202-207, 2012.

[16] Y. Wang, Z. Xiao, X. Liu, and M. Berk, "Venlafaxine modulates depression-induced behaviour and the expression of Bax mRNA and Bcl-xl mRNA in both hippocampus and myocardium," Human Psychopharmacology, vol. 26, no. 2, pp. 95-101, 2011.

[17] R. V. L. Contarteze, F. D. B. Manchado, C. A. Gobatto, and M. A. R. de Mello, "Stress biomarkers in rats submitted to swimming and treadmill running exercises," Comparative Biochemistry and Physiology A: Molecular and Integrative Physiology, vol. 151, no. 3, pp. 415-422, 2008.

[18] W. Liu, H. Sheng, Y. Xu, Y. Liu, J. Lu, and X. Ni, "Swimming exercise ameliorates depression-like behavior in chronically 
stressed rats: relevant to proinflammatory cytokines and IDO activation," Behavioural Brain Research, vol. 242, no. 1, pp. 110116, 2013.

[19] P. Jiang, W.-Y. Zhang, H.-D. Li, H.-L. Cai, Y.-P. Liu, and L.Y. Chen, "Stress and vitamin D: altered vitamin D metabolism in both the hippocampus and myocardium of chronic unpredictable mild stress exposed rats," Psychoneuroendocrinology, vol. 38, no. 10, pp. 2091-2098, 2013.

[20] P. C. Casarotto and R. Andreatini, "Repeated paroxetine treatment reverses anhedonia induced in rats by chronic mild stress or dexamethasone," European Neuropsychopharmacology, vol. 17, no. 11, pp. 735-742, 2007.

[21] Z. Radak, A. Toldy, Z. Szabo et al., "The effects of training and detraining on memory, neurotrophins and oxidative stress markers in rat brain," Neurochemistry International, vol. 49, no. 4, pp. 387-392, 2006.

[22] Z. Z. Meng, J. X. Chen, Y. M. Jiang, and H. T. Zhang, "Effect of xiaoyaosan decoction on learning and memory deficit in rats induced by chronic immobilization stress," Evidence-Based Complementary and Alternative Medicine, vol. 2013, Article ID 297154, 8 pages, 2013.

[23] E. R. Drzyzga, A. Marcinowska, and E. Obuchowicz, "Antiapoptotic and neurotrophic effects of antidepressants: a review of clinical and experimental studies," Brain Research Bulletin, vol. 79, no. 5, pp. 248-257, 2009.

[24] J. Maniam and M. J. Morris, "Voluntary exercise and palatable high-fat diet both improve behavioural profile and stress responses in male rats exposed to early life stress: role of hippocampus," Psychoneuroendocrinology, vol. 35, no. 10, pp. 1553-1564, 2010.

[25] J. Alder, S. Thakker-Varia, D. A. Bangasser et al., "Brain-derived neurotrophic factor-induced gene expression reveals novel actions of VGF in hippocampal synaptic plasticity," Journal of Neuroscience, vol. 23, no. 34, pp. 10800-10808, 2003.

[26] Y. Bian, Z. Pan, Z. Hou, C. Huang, W. Li, and B. Zhao, "Learning, memory, and glial cell changes following recovery from chronic unpredictable stress," Brain Research Bulletin, vol. 88, no. 5, pp. 471-476, 2012.

[27] T. Lee, J. Saruta, K. Sasaguri, S. Sato, and K. Tsukinoki, "Allowing animals to bite reverses the effects of immobilization stress on hippocampal neurotrophin expression," Brain Research, vol. 1195, pp. 43-49, 2008.

[28] Q. Liu, H.-Y. Zhu, B. Li, Y.-Q. Wang, J. Yu, and G.-C. $\mathrm{Wu}$, "Chronic clomipramine treatment restores hippocampal expression of glial cell line-derived neurotrophic factor in a rat model of depression," Journal of Affective Disorders, vol. 141, no. 2-3, pp. 367-372, 2012.

[29] K. Y. Zhu, Q.-Q. Mao, S.-P. Ip et al., "A standardized chinese herbal decoction, kai-xin-san, restores decreased levels of neurotransmitters and neurotrophic factors in the brain of chronic stress-induced depressive rats," Evidence-Based Complementary and Alternative Medicine, vol. 2012, Article ID 149256, 13 pages, 2012.

[30] T. Kiuchi, H. Lee, and T. Mikami, "Regular exercise cures depression-like behavior via VEGF-Flk-1 signaling in chronically stressed mice," Neuroscience, vol. 207, pp. 208-217, 2012.

[31] J. L. Trejo, M. V. LLorens-Martín, and I. Torres-Alemán, “The effects of exercise on spatial learning and anxiety-like behavior are mediated by an IGF-I-dependent mechanism related to hippocampal neurogenesis," Molecular and Cellular Neuroscience, vol. 37, no. 2, pp. 402-411, 2008.
[32] K. Fabel, K. Fabel, B. Tam et al., "VEGF is necessary for exerciseinduced adult hippocampal neurogenesis," European Journal of Neuroscience, vol. 18, no. 10, pp. 2803-2812, 2003.

[33] J. L. Trejo, E. Carro, and I. Torres-Alemán, “Circulating insulinlike growth factor I mediates exercise-induced increases in the number of new neurons in the adult hippocampus," The Journal of Neuroscience, vol. 21, no. 5, pp. 1628-1634, 2001.

[34] A. Bachis, M. I. Cruz, R. L. Nosheny, and I. Mocchetti, "Chronic unpredictable stress promotes neuronal apoptosis in the cerebral cortex," Neuroscience Letters, vol. 442, no. 2, pp. 104-108, 2008.

[35] T. A. Kosten, M. P. Galloway, R. S. Duman, D. S. Russell, and C. D'Sa, "Repeated unpredictable stress and antidepressants differentially regulate expression of the Bcl-2 family of apoptotic genes in rat cortical, hippocampal, and limbic brain structures," Neuropsychopharmacology, vol. 33, no. 7, pp. 1545-1558, 2008. 


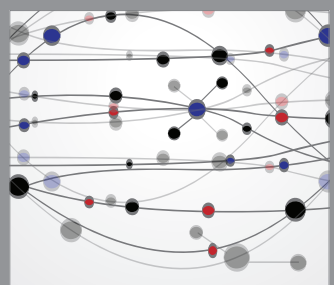

The Scientific World Journal
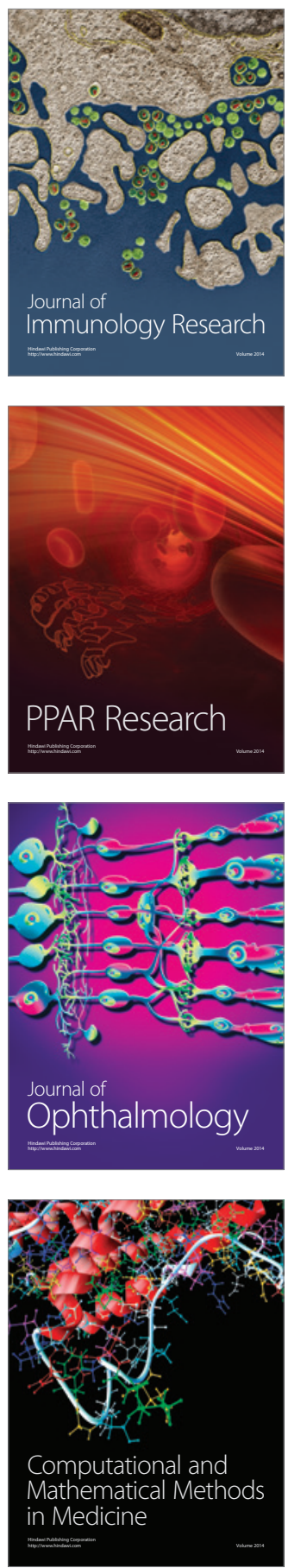

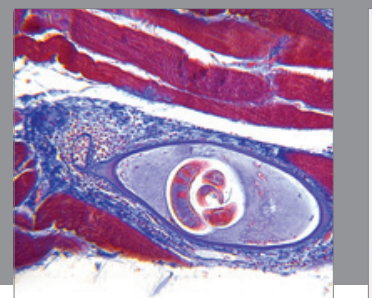

Gastroenterology

Research and Practice
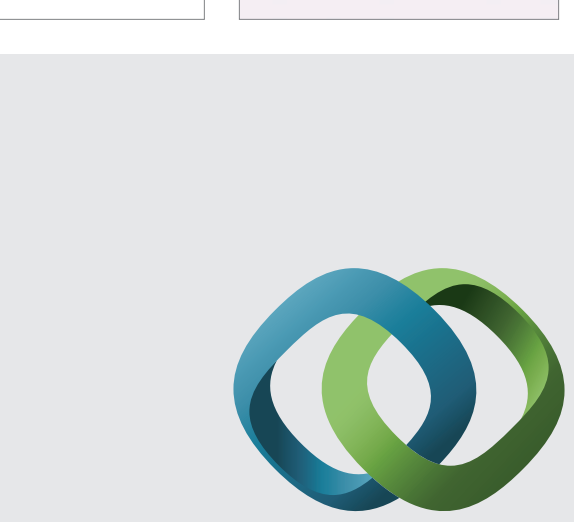

\section{Hindawi}

Submit your manuscripts at

http://www.hindawi.com
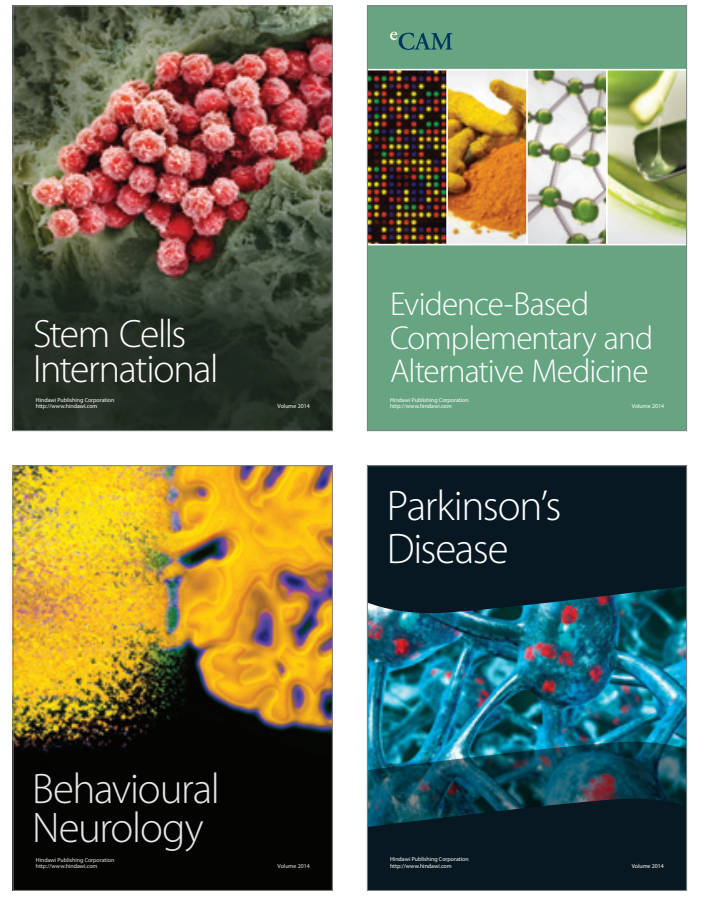
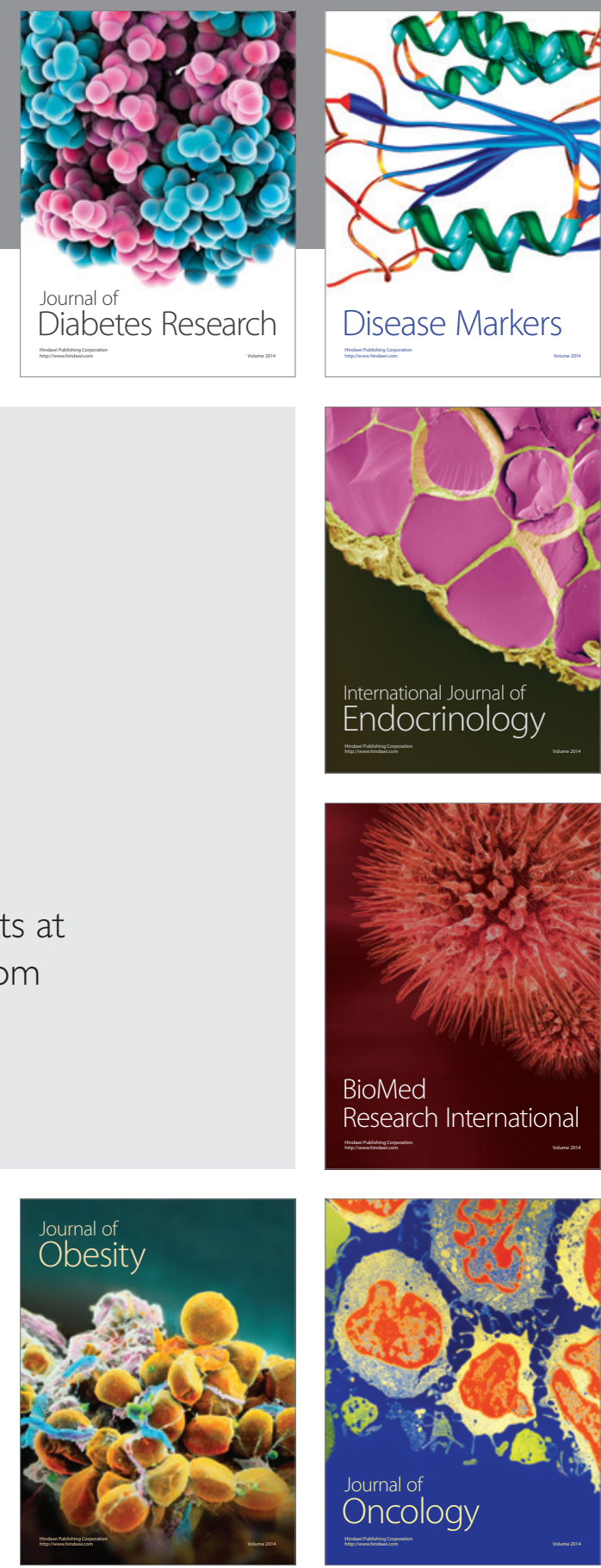

Disease Markers
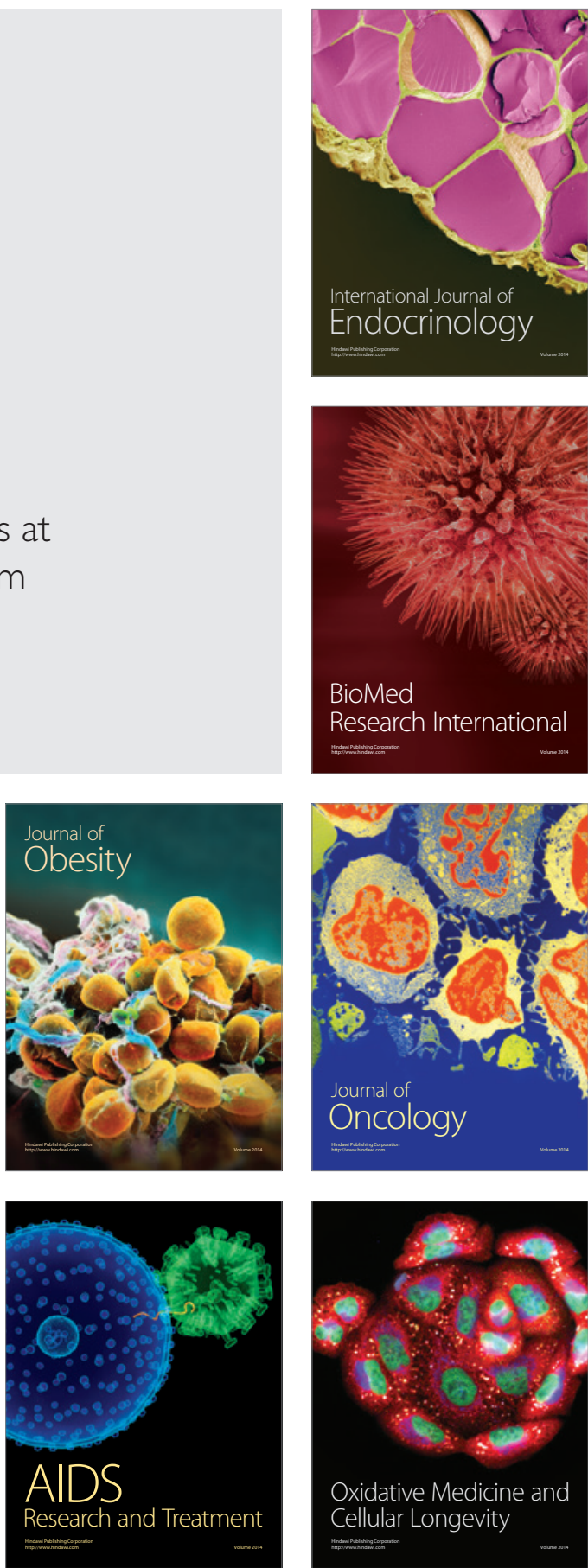